\title{
Entropic proofs of Singleton bounds for quantum error-correcting codes
}

\author{
Markus Grassl $^{\circledR}$, Senior Member, IEEE, Felix Huber ${ }^{\circledR}$, and Andreas Winter ${ }^{\circledR}$
}

\begin{abstract}
We show that a relatively simple reasoning using von Neumann entropy inequalities yields a robust proof of the quantum Singleton bound for quantum errorcorrecting codes (QECC). For entanglement-assisted quantum error-correcting codes (EAQECC) and catalytic codes (CQECC), a type of generalized quantum Singleton bound [Brun et al., IEEE Trans. Inf. Theory 60(6):3073-3089 (2014)] was believed to hold for many years until recently one of us found a counterexample [MG, Phys. Rev. A 103, 020601 (2021)]. Here, we rectify this state of affairs by proving the correct generalized quantum Singleton bound, extending the above-mentioned proof method for QECC; we also prove information-theoretically tight bounds on the entanglementcommunication tradeoff for EAQECC. All of the bounds relate block length $n$ and code length $k$ for given minimum distance $d$ and we show that they are robust, in the sense that they hold with small perturbations for codes which only correct most of the erasure errors of less than $d$ letters. In contrast to the classical case, the bounds take on qualitatively different forms depending on whether the minimum distance is smaller or larger than half the block length. We also provide a propagation rule: any pure QECC yields an EAQECC with the same distance and dimension, but of shorter block length.
\end{abstract}

Index Terms-Quantum codes, quantum entanglement, Singleton bound.

\section{INTRODUCTION}

$\mathbf{T}$ HE object of the present paper are quantum errorcorrecting codes (QECC), plain and with entanglement assistance. The general communication diagramme is shown in Fig. 1: a $K$-dimensional system $M$ (the "message") is encoded into $n$ quantum systems $X_{1}, \ldots, X_{n}$ having $q$ levels each. (Note that we do not restrict $q$ to be power of prime). This is assisted by entanglement $|\varphi\rangle^{A_{i n} B_{i n}}$ at the input, which is quantified by the entanglement entropy $\ell_{i n}=S\left(A_{i n}\right)_{\varphi}=S\left(\varphi^{A_{i n}}\right)=S\left(B_{i n}\right)_{\varphi}$, where we denote by $\varphi^{S}$ the marginal state of $\varphi$ on some system $S$. A QECC of minimum distance $d$ can correct

Markus Grassl is with the International Centre for Theory of Quantum Technologies, University of Gdansk, 80-308 Gdańsk, Poland.

Felix Huber was with ICFO - Institut de Ciències Fotòniques, ES 08860 Castelldefels (Barcelona), Spain, and is now with the Atomic Optics Department, Jagiellonian University, 30-348 Kraków, Poland.

Andreas Winter is with the Departament de Física: Grup d'Informació Quàntica, Universitat Autònoma de Barcelona, ES-08193 Bellaterra (Barcelona), Spain, as well as with ICREA - Institució Catalana de Recerca i Estudis Avançats, Pg. Lluis Companys, 23, 08010 Barcelona, Spain.

This paper was presented at Beyond IID in Information Theory 8 , Stanford, 9-13 November 2020. up to $d-1$ erasures [1]. In this case, the quantum erasure channel partitions the $n$ input systems $X_{1} \ldots X_{n}$ into two disjoint blocks $X_{I}$ and $X_{J}$ of size $|I|=n-d+1$ and $|J|=d-1$, respectively. The decoder receives only the block $X_{I}$, together with classical information on the index set $I \subset\{1, \ldots, n\}=[n]$. The perfect decoding of the quantum information $M$ on the output system $\widehat{M}$ is equivalent to reproducing the maximally entangled state $\left|\Phi_{K}\right\rangle^{M R}$ between $\widehat{M}$ and $R$ (i.e. $\left|\Phi_{K}\right\rangle^{\widehat{M} R}$ ) with fidelity 1 , where $R$ is a reference system. Additionally, we allow the recovery of some of the entanglement between the subsystems $A_{f}$ and $B_{f}$ in a pure state $\left|\varphi^{\prime}\right\rangle^{A_{f} B_{f}}$, which is quantified as $\ell_{f}=S\left(A_{f}\right)_{\varphi^{\prime}}$. The net consumption $\ell:=\ell_{i n}-\ell_{f}$ of entanglement can thus be positive, negative, or zero. The amount of quantum information encoded into $M$ is $\log _{2} K$. Note that in information theory, $\log$ is usually understood to be the binary logarithm, in particular in the von Neumann entropy $S(\rho)=-\operatorname{Tr} \rho \log _{2} \rho$. This convention means that the quantum information is counted in units of qubits, and the entanglement in units of ebits. However, in coding theory, assuming that all $X_{i}$ are $q$-dimensional, the $q$-ary logarithm is preferred. To avoid confusion, we will include the base in the notation for the logarithm throughout.

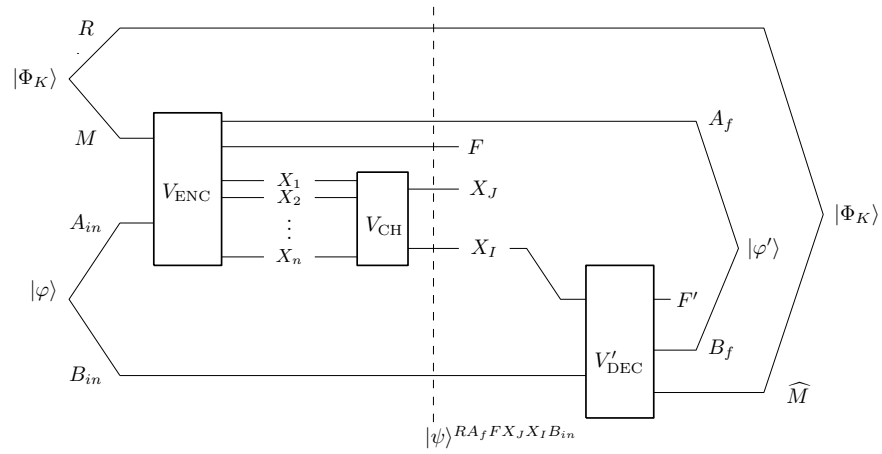

Fig. 1. Diagramme of a general entanglement-assisted quantum error-correcting code (EAQECC) as a quantum transmission and entanglement-generation procedure between the encoder $V_{\text {ENC }}$ and the decoder $V_{\mathrm{DEC}}^{\prime}$. Both encoder and decoder are written as isometries, with auxiliary output systems $F$ and $F^{\prime}$, respectively. The erasure channel $V_{\mathrm{CH}}$ partitions the systems $X_{1} X_{2} \ldots X_{n}$ into two disjoint blocks $X_{I}$ and $X_{J}$ with $I, J \subset[n]$ that are of size $n-d+1$ and $d-1$ respectively. The block $X_{J}$ is erased, while the block $X_{I}$, together with information about the index set $I$, is received by the decoder. We usually assume that all $X_{i}$ have the same dimension $q$. 
One of the basic bounds in coding theory is the Singleton bound [2]. It has a well-known quantum analogue due to Knill and Laflamme [3], upper bounding the dimension $K$ in the absence of any prior entanglement (see also [4], [5]). For an unassisted code, i. e. $\ell_{i n}=0$, it states in our notation that

$$
\log _{q} K \leq n-2 d+2,
$$

which in particular means that $d-1<\frac{n}{2}$ is necessary for any non-trivial code with $K>1$ to exist (indeed, this holds due to the familiar no-cloning argument [6]). We start by reviewing a proof of the quantum Singleton bound that uses a simple entropic reasoning [7], and that gives a potentially tighter entropic bound for unassisted codes than the one previously known [3], [4]. The bulk of the paper is however concerned with entanglementassisted codes [8], for which we generalize this first proof.

In actual codes it will often be the case that $K$ is a power of 2 (or of $q$ ), and also $|\varphi\rangle,\left|\varphi^{\prime}\right\rangle,|\Phi\rangle$ will be maximally entangled states of Schmidt rank a power of 2 (or of $q$ ). In that case, $\log _{2} K$ counts the number of qubits encoded; similarly, $\ell_{i n}$ and $\ell_{f}$ count the initial and final number of EPR pairs (ebits), respectively. But to achieve the largest generality of our bounds, which state necessary conditions for the existence of codes, we shall make no such assumption and none of $\log _{2} K, \log _{q} K$, $\ell_{i n}$, and $\ell_{f}$ needs to be an integer.

Since the code can transmit $\log _{2} K$ qubits from Alice to Bob, it can also be used to distribute $\log _{2} K$ ebits of entanglement. In fact, in the communication diagramme, it would be Alice to prepare the maximally entangled Bell state $\left|\Phi_{K}\right\rangle^{R M}$, keeping $R$ and encoding $M$. This shifts the focus from sending qubits to generating entanglement between the parties: starting from an initial state $|\varphi\rangle^{A_{i n} B_{i n}}$ and ending with $\left|\Phi_{K}\right\rangle^{R \widehat{M}} \otimes\left|\varphi^{\prime}\right\rangle^{A_{f} B_{f}}$, the net entanglement generated is $\log _{2} K-\ell$ ebits. This is the point of view of catalytic quantum error correction [9]. It allows us to simplify the communication diagramme: the generation of $\left|\Phi_{K}\right\rangle$ becomes part of the encoding isometry, while renaming $A_{f} R$ as $A_{f}$ and $B_{f} \widehat{M}$ as $B_{f}$; we thus arrive at Fig. 2. This procedure turns any entanglementassisted quantum error-correcting codes (EAQECC) for $\log _{2} K$ qubits and using $\ell$ ebits of entanglement into a catalytic quantum error-correcting code (CQECC) with net generation of $\log _{2} K-\ell$ ebits. Naturally, for such catalytic code to be useful one wants $\log _{2} K-\ell>0$.

The rest of the paper is structured as follows: In Section II, as a warm-up we re-derive the quantum Singleton bound for subspace codes by a simple entropic reasoning. In Section III we show that its proof generalizes to a proof of a corrected version of the originally claimed quantum Singleton bound for general EAQECCs and CQECCs, while in Section IV, we prove tight tradeoff relations between encoded quantum information and net entanglement consumption in general EAQECCs. In Section $\mathrm{V}$ we present constructions, for sufficiently

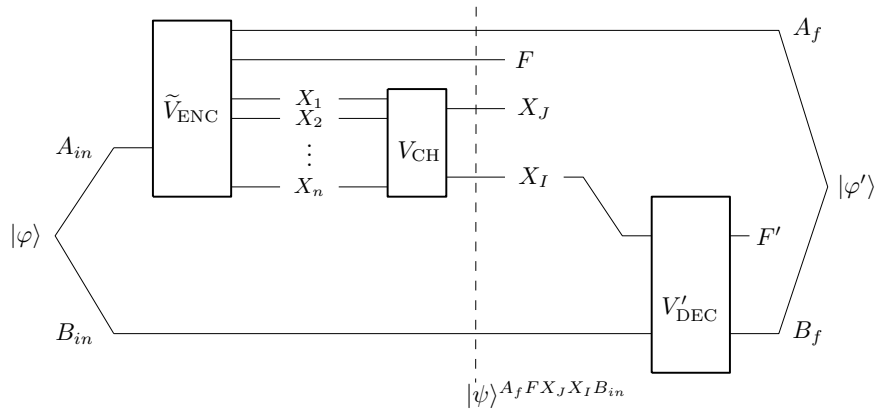

Fig. 2. Diagramme of a general entanglement-assisted quantum errorcorrecting code (EAQECC) as an entanglement-generation procedure between the encoder and the decoder. Starting from Fig. 1, we simply integrate the creation of the maximally entangled state $\left|\Phi_{K}\right\rangle^{R M}$ into the encoding isometry $\widetilde{V}_{\mathrm{ENC}}$ and merge $R$ and $A_{f}$ to get a larger register for the generated entanglement.

large alphabet size, showing the tightness of the derived bounds, and conclude in Section VI.

\section{SINGLETON BOUND FOR ISOMETRIC-ENCODING QECC}

We start with the usual setting of QECC as subspaces of the $n$-party system $X_{1} X_{2} \ldots X_{n}$, where the encoder does not share prior entanglement. That is, in Figs. 1 and 2, the subsystems $A_{i n}, B_{i n}$ and $F$ are trivial $(F=$ $A_{\text {in }}=B_{\text {in }}=\mathbb{C}$ ).

Define by

$$
\bar{\sigma}=\frac{1}{d-1} \mathbb{E}_{|J|=d-1} S\left(X_{J}\right) \leq \log _{2} q
$$

the average entropy of a random $(d-1)$-block per system. Note that in keeping with coding convention, we will from now on focus on situations where all systems are $q$-ary.

Theorem 1 (Entropic quantum Singleton bound [7])

Let $\mathcal{Q}$ be a subspace of $X_{1} X_{2} \ldots X_{n}$ that corresponds to a QECC $((n, K, d))_{q}$ of dimension $K>1$ and distance $d$. Then,

$$
\begin{aligned}
\log _{2} K & \leq \max \{0, n-2 d+2\} \bar{\sigma} \\
& \leq \max \{0, n-2 d+2\} \log _{2} q .
\end{aligned}
$$

Proof: Purify the maximally mixed state on the code space, $\rho=\frac{1}{K} \Pi_{Q}$ where $\Pi_{Q}$ is the projector onto the code space, with a reference system $R$ of dimension $K$, and go to the entanglement-generating code picture (thus, $A_{\text {in }}=$ $B_{i n}=\mathbb{C}$ in Fig. 1 , or equivalently $R \equiv A_{f}$ in Fig. 2, while $\left.A_{i n}=B_{i n}=\mathbb{C}\right)$. The condition for perfect quantum error correction is equivalent, for a code of distance $d$, to the relation

$$
S\left(X_{I}\right)=S\left(R X_{J}\right)=S(R)+S\left(X_{J}\right)
$$

for any bipartition $[n]=I \dot{U} J$ into disjoint subsets of cardinalities $|I|=n-d+1$ and $|J|=d-1$, respectively; cf. [10]. Indeed, if Eq. (4) holds, then we can apply the decoding isometry $V^{\prime}: X_{I} \rightarrow B_{f} F^{\prime}$ in order to recover 
the pure entangled state between $R$ and the first output register $B_{f}$ of the isometry, whereas the other output register $F^{\prime}$ is left in a pure entangled state with $F X_{J}$. Taking averages over partitions, we get

$$
S(R)=\mathbb{E}_{|I|=n-d+1} S\left(X_{I}\right)-\mathbb{E}_{|J|=d-1} S\left(X_{J}\right) .
$$

Here, $\mathbb{E}_{I} S\left(X_{I}\right)$ is the average von Neumann entropy of subsystems with size $|I|=n-d+1$. We now make use of Eq. (7) from Lemma 2 below. Choosing $m=n-d+1$ and $\mu=d-1$, one has then

$$
\begin{aligned}
S(R) & =\mathbb{E}_{|I|=n-d+1} S\left(X_{I}\right)-\mathbb{E}_{|J|=d-1} S\left(X_{J}\right) \\
& \leq\left(\frac{n-d+1}{d-1}-1\right) \mathbb{E}_{|J|=d-1} S\left(X_{J}\right) .
\end{aligned}
$$

This proves the claim. Note that we could apply the lemma because it is known that any nontrivial code of distance $d$ must satisfy $d-1<\frac{n}{2}$.

Note that the entropic Singleton bound (3) takes the average entropy (2) of a random subblock of size $d-1$ of the code into account. Thereby, it refines the bound $\log _{2} K \leq(n-2 d+2) \log _{2} q$.

Lemma 2 Consider the n-party system $X_{1} X_{2} \ldots X_{n}$, and for a subset $I \subset[n]$ of the ground set denote $X_{I}=\bigotimes_{i \in I} X_{i}$. Let $\mu<m \leq n$. Then, with respect to any state $\rho$,

$$
\mathbb{E}_{|I|=m} S\left(X_{I}\right) \leq \frac{m}{\mu} \mathbb{E}_{|J|=\mu} S\left(X_{J}\right),
$$

where both expectation values are with respect to uniformly random subsets $I, J \subset[n]$ of the ground set, of the respective cardinality.

This lemma appears to have been stated first in [11], albeit with an incorrect proof; then again in [12], with a proof attributed to AW; see [13] for a generalized perspective. For later use we will actually prove directly a generalization of it to the conditional entropy:

Lemma 3 Consider an $(n+1)$-party system $X_{1} X_{2} \ldots X_{n} Y$ and denote $X_{I}=\bigotimes_{i \in I} X_{i}$ for any subset $I \subset[n]$. Let $\mu<$ $m \leq n$. Then, with respect to any state $\rho$,

$$
\mathbb{E}_{|I|=m} S\left(X_{I} \mid Y\right) \leq \frac{m}{\mu} \mathbb{E}_{|J|=\mu} S\left(X_{J} \mid Y\right),
$$

where both expectation values are with respect to uniformly random subsets $I, J \subset[n]$ of the respective cardinality.

Proof: We start with the case $n=m>\mu=n-1$. For this, we have to show that

$$
\begin{aligned}
S\left(X_{[n]} \mid Y\right) & \leq \frac{n}{n-1} \mathbb{E}_{J} S\left(X_{J} \mid Y\right) \\
& =\frac{1}{n-1} \sum_{i=1}^{n} S\left(X_{[n] \backslash\{i\}} \mid Y\right) .
\end{aligned}
$$

Purifying the state to a pure state $|\psi\rangle^{X_{[n]} Y Z}$ with an auxiliary system $Z$, we can rewrite the left hand side as $S\left(X_{[n]} \mid Y\right)=S(Z)-S(Y)$, and the terms in the sum on the right hand side as $S\left(X_{[n] \backslash\{i\}} \mid Y\right)=S\left(X_{i} Z\right)-S(Y)$, making Eq. (9) equivalent to

$$
(n-1) S(Z)-(n-1) S(Y) \leq \sum_{i=1}^{n} S\left(X_{i} Z\right)-n S(Y) \text {. }
$$

Adding $n S(Y)$ and subtracting $n S(Z)$ from both sides, this becomes equivalent to

$$
\begin{aligned}
S(Y)-S(Z) & =S\left(X_{[n]} Z\right)-S(Z) \\
& =S\left(X_{[n]} \mid Z\right) \leq \sum_{i=1}^{n} S\left(X_{i} \mid Z\right),
\end{aligned}
$$

which is indeed true by strong subadditivity.

Repeated application of Eq. (9) leads now, for each $I \subset[n]$ with $|I|=m$, to

$$
S\left(X_{I} \mid Y\right) \leq \frac{m}{\mu} \mathbb{E}_{J \subset I,|J|=\mu} S\left(X_{J} \mid Y\right)
$$

Taking the average over all subsets $I$ concludes the proof.

A code that saturates the quantum Singleton bound from Theorem 1 with equality is called quantum minimum distance separable (QMDS). Taking advantage of the above entropic proof, we can derive some interesting properties of such codes. To state the following corollary, recall that a code of distance $d$ is called pure if $\langle\phi|E| \phi\rangle=0$ holds for all code states $|\phi\rangle$ and for all traceless errors $E$ of weight smaller than $d$. This is equivalent to every code state having maximally mixed $(d-1)$-body marginals.

Corollary 4 (Cf. [7]) Let $\mathcal{Q}=((n, K, d))_{q}$ be a $Q M D S$ code. Then it is pure.

Proof: For a QMDS code, $S(R)=\log _{2} K$. Thus to satisfy Eq. (6), $S\left(X_{J}\right)=(d-1) \log _{2} q$ for all $J \subset[n]$ of size $d-1$ must hold. This proves the claim.

Thus, we can make a statement about the entanglement that is necessary for QMDS codes to exist: Any code state necessarily needs to have the same $(d-1)$ marginals as the maximally mixed state on the code subspace. Consequently any code state of a QMDS code is maximally entangled across each $d-1$ vs. $n-d+1$ bipartition. Vice versa, if the code states are less entangled, it means that $\bar{\sigma}$ is less than its maximum value $\log _{2} q$, and that additionally limits the amount of information that can be encoded.

\section{QUANTUM SINGLETON BOUND FOR GENERAL EAQECC}

We now show that the above ideas carry over to the analysis of EAQECCs. We start with a bound on the net entanglement generation of such codes, which is most naturally discussed in the setting of CQECCs.

\section{Theorem 5 (Catalytic quantum Singleton bound)}

Consider a catalytic entanglement generation code as in Fig. 2 with net entanglement production $\ell_{f}-\ell_{i n}$. Then, the 
latter is bounded by the quantum Singleton bound. Namely, with respect to the state $|\psi\rangle^{A_{f} F X_{J} X_{I} B_{i n}}$ in Fig. 2, one has

$$
\begin{aligned}
\ell_{f}-\ell_{i n} & =S\left(A_{f}\right)-S\left(B_{i n}\right) \\
& \leq \max \{0,(n-2 d+2) \bar{\sigma}\} \\
& \leq \max \{0, n-2 d+2\} \log _{2} q .
\end{aligned}
$$

Here, $S\left(B_{i n}\right)_{\varphi}=S\left(B_{i n}\right)_{\psi}$ and $S\left(A_{f}\right)_{\varphi^{\prime}}=S\left(A_{f}\right)_{\psi}$ are the initial and final entanglement, respectively. As before, $\bar{\sigma}=$ $\frac{1}{d-1} \mathbb{E}_{|J|=d-1} S\left(X_{J}\right) \leq \log _{2} q$ is the average entropy of $a(d-$ 1)-block per system.

Proof: By assumption, there is a decoding isometry $V^{\prime}: X_{I} B_{i n} \rightarrow B_{f} F^{\prime}$ that maps $|\psi\rangle^{A_{f} F X_{I} X_{J} B_{i n}}$ to

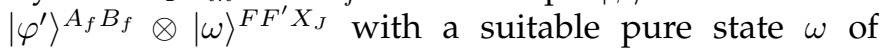
$F F^{\prime} X_{J}$. Thus,

$$
\begin{aligned}
S\left(X_{I} B_{i n}\right)_{\psi} & =S\left(B_{f}\right)_{\varphi^{\prime}}+S\left(F^{\prime}\right)_{\omega} \\
& =S\left(A_{f}\right)_{\varphi^{\prime}}+S\left(F X_{J}\right)_{\omega} \\
& =S\left(A_{f}\right)_{\psi}+S\left(F X_{J}\right)_{\psi} \\
& =S\left(A_{f}\right)_{\psi}+S\left(X_{J}\right)_{\psi}+S\left(F \mid X_{J}\right)_{\psi},
\end{aligned}
$$

using the purity of the states $\varphi^{\prime}$ and $\omega$. On the other hand, by subadditivity of the von Neumann entropy,

$$
S\left(X_{I} B_{i n}\right)_{\psi} \leq S\left(X_{I}\right)_{\psi}+S\left(B_{i n}\right)_{\psi}
$$

yielding

$$
S\left(A_{f}\right)-S\left(B_{i n}\right) \leq S\left(X_{I}\right)-S\left(X_{J}\right)-S\left(F \mid X_{J}\right) .
$$

By taking the average over all partitions $[n]=I \dot{\cup} J$ into blocks of size $n-d+1$ and $d-1$, respectively, we get the basic bound

$$
\begin{array}{r}
S\left(A_{f}\right)-S\left(B_{i n}\right) \leq \mathbb{E}_{|I|=n-d+1} S\left(X_{I}\right)-\mathbb{E}_{|J|=d-1} S\left(X_{J}\right) \\
-\mathbb{E}_{|J|=d-1} S\left(F \mid X_{J}\right) .
\end{array}
$$

Let us start with the case $d-1<n-d+1$. We can then apply Lemma 2 with $m=n-d+1$ and $\mu=d-1$, giving $\mathbb{E}_{|I|=n-d+1} S\left(X_{I}\right) \leq \frac{n-d+1}{d-1} \mathbb{E}_{|J|=d-1} S\left(X_{J}\right)$, hence

$$
\begin{aligned}
\ell_{f}-\ell_{i n} & =S\left(A_{f}\right)-S\left(B_{i n}\right) \\
\leq & \frac{n-2 d+2}{d-1} \mathbb{E}_{|J|=d-1} S\left(X_{J}\right) \\
& -\mathbb{E}_{|J|=d-1} S\left(F \mid X_{J}\right) .
\end{aligned}
$$

The first term on the r.h.s. equals $(n-2 d+2) \bar{\sigma}$, and we will show that the other expectation value is nonnegative. Indeed,

$$
\begin{aligned}
\mathbb{E}_{|J|=d-1} S\left(F \mid X_{J}\right) & \\
& =\frac{1}{2} \mathbb{E}_{\substack{|J|=\left|J^{\prime}\right|=d-1 \\
J \cap J^{\prime}=\emptyset}}\left(S\left(F \mid X_{J}\right)+S\left(F \mid X_{J^{\prime}}\right)\right) \geq 0,
\end{aligned}
$$

the latter because $S\left(F \mid X_{J}\right)+S\left(F \mid X_{J^{\prime}}\right) \geq 0$ by strong subadditivity.

It remains to show $S\left(A_{f}\right)-S\left(B_{i n}\right) \leq 0$ when $d-1 \geq$ $n-d+1$. Going back to Eq. (17), we reproduce the r.h.s. by decomposing system $J$ as $J=J_{1} \dot{\cup} J_{2}$, with $\left|J_{1}\right|=n-$ $d+1$ and $\left|J_{2}\right|=2(d-1)-n$. We can then write

$$
\begin{aligned}
\mathbb{E}_{|J|=d-1}\left(S\left(X_{J}\right)+S\left(F \mid X_{J}\right)\right) & =\mathbb{E}_{J_{1}, J_{2}} S\left(F X_{J_{1}} X_{J_{2}}\right) \\
& =\mathbb{E}_{I, J_{2}} S\left(F X_{I} X_{J_{2}}\right),
\end{aligned}
$$

and so we get

$$
\begin{aligned}
\ell_{f}-\ell_{i n} & =S\left(A_{f}\right)-S\left(B_{i n}\right) \\
& \leq-\mathbb{E}_{I, J_{2}} S\left(F X_{J_{2}} \mid X_{I}\right) \\
& =-\mathbb{E}_{J_{1}, J_{2}} S\left(F X_{J_{2}} \mid X_{J_{1}}\right),
\end{aligned}
$$

where the expectations are with respect to uniformly random partitions $[n]=I \dot{\cup} J_{1} \cup J_{2}$ with $|I|=\left|J_{1}\right|=n-$ $d+1$ and $\left|J_{2}\right|=2(d-1)-n$. We can now again argue with strong subadditivity: $S\left(F X_{J_{2}} \mid X_{I}\right)+S\left(F X_{J_{2}} \mid X_{J_{1}}\right) \geq 0$, and so the right hand side of the previous displayed equation is non-positive.

Theorem 5 provides the quantum Singleton bound in the most general setting of catalytic entanglement generation and without restrictions on the encoder, apart from being given by a completely positive trace-preserving (cptp) map. Even in the non-assisted setting it thus goes beyond the original assumptions of Knill and Laflamme [3], and of Rains [4], who treated stabilizer and general subspace codes.

It is interesting to reflect on the status of the bound $d-1<\frac{n}{2}$, which holds in the non-assisted case due to no-cloning (cf. the original quantum Singleton bound), and obviously requires a non-trivial code of dimension larger than 1. We note that this bound is no longer true in the case of EAQECC as a counterexample provided in Refs. [14], [15] shows. Nevertheless, the above theorem still gives it a meaning, because it states that $\log _{K}-\ell \leq 0$ holds when $d-1 \geq \frac{n}{2}$ and thus the code cannot be used to generate any net entanglement. The analogous (and well-known) Shannon-theoretic statement is that not only can the 50-50 erasure channel not transmit any quantum information, or for that matter generate entanglement, but it cannot even be used to increase the amount of entanglement given any pre-shared entanglement.

The bound for the generation of net entanglement is clearly the best possible: If $d-1 \geq \frac{n}{2}$ then $\ell_{f}-\ell_{i n}$ can reach at most 0 . In this case we can always recover the initial entanglement fully at the end by simply not touching it; if $d-1<\frac{n}{2}$ on the other hand, it is known that for sufficiently large $q$ there are QMDS codes saturating the bound when $k=\log _{q} K=n-2 d+2$, in which case there is no need for any entanglement assistance.

\section{TRANSMISSION-ENTANGLEMENT TRADEOFF}

The net entanglement generation is only part of the story, since the bound of Theorem 5 conflates the qubits sent through the code with the entanglement consumed by it. To see why that happens, consider the extreme case when unlimited entanglement can be consumed, i. e. $\ell$ is 
unbounded. We can then beat the unassisted quantum Singleton bound by the following entanglement-assisted scheme: using dense coding for each of the $q$-ary quantum systems (requiring $n$ maximally entangled states of Schmidt rank $q$ ) we can turn each of the $n$ quantum channels of $\log _{2} q$ qubits into classical channels of $\log _{2} q^{2}$ classical bits. As $d-1$ of these systems undergo erasure, we can use a classical MDS code for alphabet size $q^{2}$, encoding $(n-d+1) \log _{2} q^{2}$ messages. These messages in turn are used to teleport $n-d+1 q$-ary quantum systems, requiring another $(n-d+1) \log _{2} q$ ebits. Thus, we get $k=(n-d+1) \log _{2} q$, saturating the classical Singleton bound! Note that the entanglement consumption attaining the same code size can be reduced to $(d-1) \log _{2} q$ ebits; see the discussion after the main theorem of this section.

Next, we derive the inequalities for the full tradeoff between the transmitted quantum information $\log _{2} K$ and the consumed entanglement $\ell$. Define by

$$
\begin{aligned}
& \bar{\sigma}:=\frac{1}{d-1} \mathbb{E}_{|J|=d-1} S\left(X_{J}\right) \leq \log _{2} q \\
& \overline{\bar{\sigma}}:=\frac{1}{n-d+1} \mathbb{E}_{|I|=n-d+1} S\left(X_{I}\right) \leq \log _{2} q
\end{aligned}
$$

the average entropies of a $(d-1)$-block and an $(n-d+$ 1)-block per system respectively. We have the following theorem on the qubit vs. ebit (i. e., $\log _{2} K$ vs. $\ell$ ) tradeoff.

Theorem 6 (Qubit-ebit Singleton bound) Consider an $E A Q E C C$ with minimum distance $d$ that encodes a $K$ dimensional quantum system into $n$ q-ary quantum systems and whose net entanglement consumption is $\ell=\ell_{i n}-\ell_{f}$ ebits, as illustrated in Fig. 1. Then the following bounds hold. If $d-1<\frac{n}{2}$, then

$$
\begin{aligned}
& \log _{2} K \leq(n-2 d+2) \bar{\sigma}+\ell, \\
& \log _{2} K \leq(n-d+1) \overline{\bar{\sigma}} \leq(n-d+1) \bar{\sigma} .
\end{aligned}
$$

$$
\text { If } \begin{aligned}
d- & 1 \geq \frac{n}{2}, \text { then } \\
& \log _{2} K \leq \ell, \\
& \log _{2} K \leq(n-d+1) \overline{\bar{\sigma}}, \\
& \log _{2} K \leq \frac{n-d+1}{3 d-3-n}(\ell+(2 d-2-n) \overline{\bar{\sigma}}) .
\end{aligned}
$$

The performance of an arbitrary EAQECC is thus bounded as

$$
\begin{aligned}
& \log _{2} K \leq \max \{0, n-2 d+2\} \log _{2} q+\ell, \\
& \log _{2} K \leq(n-d+1) \log _{2} q,
\end{aligned}
$$

and if $d-1 \geq \frac{n}{2}$, then

$$
\log _{2} K \leq \frac{n-d+1}{3 d-3-n}\left(\ell+(2 d-2-n) \log _{2} q\right) .
$$

The shape of the rate region (the diagramme of admissible pairs $\frac{\log _{2} K}{n \log _{2} q}=\frac{\log _{q} K}{n}$ and $\frac{\ell}{n \log _{2} q}$ ) is depicted in Fig. 3. It depends on whether the normalized distance $\delta=\frac{d-1}{n}$ is smaller or larger than $\frac{1}{2}$.

Proof: Eqs. (24) and (26) have already been shown in Theorem 5. The proofs of the three other Eqs. (25), (27), and (28) rest on entropic bounds that are similar to those used for Theorem 5 .

Concretely, with respect to the pure state

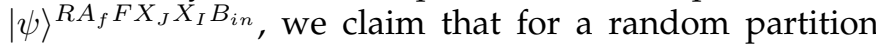
$[n]=I \cup \dot{\cup} J$ into sets of size $n-d+1$ and $d-1$, respectively:

$$
\begin{aligned}
\log _{2} K-\ell & \leq \mathbb{E}_{|I|=n-d+1,|J|=d-1}\left(S\left(X_{I}\right)-S\left(X_{J}\right)\right) \\
& =: n \Delta .
\end{aligned}
$$

Furthermore, we claim that for any partition $[n]=I \dot{\cup} J$,

$$
\begin{aligned}
2 \log _{2} K & \leq I\left(R A_{f} B_{i n} F: X_{I}\right) \\
& =S\left(X_{[n]}\right)+S\left(X_{I}\right)-S\left(X_{J}\right) \\
& =S\left(X_{I}\right)+S\left(X_{I} \mid X_{J}\right) \leq 2 S\left(X_{I}\right) .
\end{aligned}
$$

To prove Eq. (32), we first do so assuming trivial $F$. For this, it is enough to consider the situation as in Fig. 2, which we have already done in the proof of Theorem 5 . Namely, Eq. (16), with trivial $F$ system, gives precisely

$$
\log _{2} K-\ell=S\left(A_{f}\right)-S\left(B_{i n}\right) \leq S\left(X_{I}\right)-S\left(X_{J}\right),
$$

and by averaging over partitions we get, as desired,

$$
\begin{aligned}
\log _{2} K-\ell & =S\left(A_{f}\right)-S\left(B_{\text {in }}\right) \\
& \leq \mathbb{E}_{|I|=n-d+1} S\left(X_{I}\right)-\mathbb{E}_{|J|=d-1} S\left(X_{J}\right) .
\end{aligned}
$$

To prove Eq. (33), we use the data processing inequality repeatedly, as well as the fact that $R$ and $B_{i n}$ are decoupled, $I\left(R: B_{i n}\right)=0$. Then

$$
\begin{aligned}
2 \log _{2} K & =I(R: \widehat{M}) \\
& \leq I\left(R: B_{i n} X_{I}\right) \\
& =I\left(R: B_{i n}\right)+I\left(R: X_{I} \mid B_{i n}\right) \\
& \leq I\left(R B_{i n}: X_{I}\right) \\
& \leq I\left(R B_{i n} A_{f} F: X_{I}\right) .
\end{aligned}
$$

This allows to prove Eqs. (25), (27), and (28). Namely, averaging Eqs. (32) and (33) over partitions yields

$$
\begin{aligned}
2 \log _{2} K & \leq S\left(X_{[n]}\right)+n \Delta, \\
\log _{2} K & \leq \mathbb{E}_{|I|=n-d+1} S\left(X_{I}\right)=(n-d+1) \overline{\bar{\sigma}} .
\end{aligned}
$$

Now, Eq. (25) follows from (35), invoking once more Lemma 2; Eq. (27) follows from Eq. (35), too. To proof Eq. (28), we proceed as follows:

$$
\begin{aligned}
S\left(X_{[n]}\right)-S\left(X_{I}\right) & =S\left(X_{J} \mid X_{I}\right) \\
& \leq \frac{d-1}{2 d-2-n} \mathbb{E}_{\left|J^{\prime}\right|=2 d-2-n} S\left(X_{J^{\prime}} \mid X_{I}\right),
\end{aligned}
$$

using Eq. (12) with $J^{\prime} \subset J,|J|=d-1$ and $\left|J^{\prime}\right|=d-1-$ $(n-d+1)=2 d-2-n$. Taking the average over $I$ as well, we get

$$
\begin{aligned}
& S\left(X_{[n]}\right) \leq \mathbb{E}_{I} S\left(X_{I}\right)+\frac{d-1}{2 d-2-n} \mathbb{E}_{I, J^{\prime}} S\left(X_{J^{\prime}} \mid X_{I}\right) \\
& \quad=(n-d+1) \overline{\bar{\sigma}}+\frac{d-1}{2 d-2-n}\left(\mathbb{E}_{J} S\left(X_{J}\right)-\mathbb{E}_{I} S\left(X_{I}\right)\right) \\
& \quad=(n-d+1) \overline{\bar{\sigma}}-\frac{d-1}{2 d-2-n} n \Delta .
\end{aligned}
$$




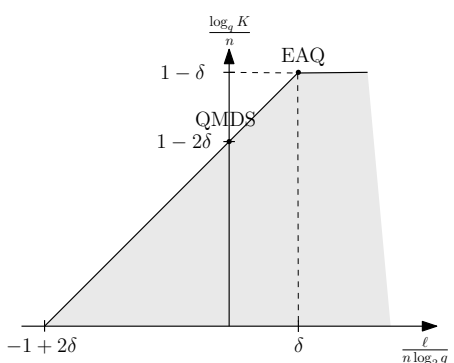

(a) $\delta=\frac{d-1}{n}<\frac{1}{2}$

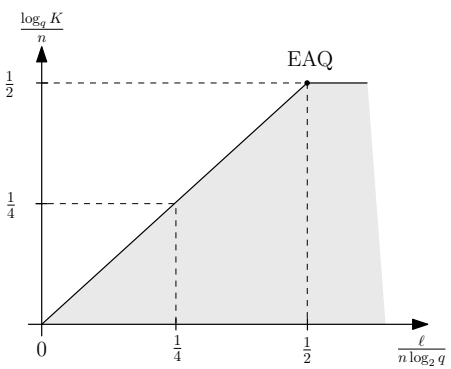

(b) $\delta=\frac{1}{2}$

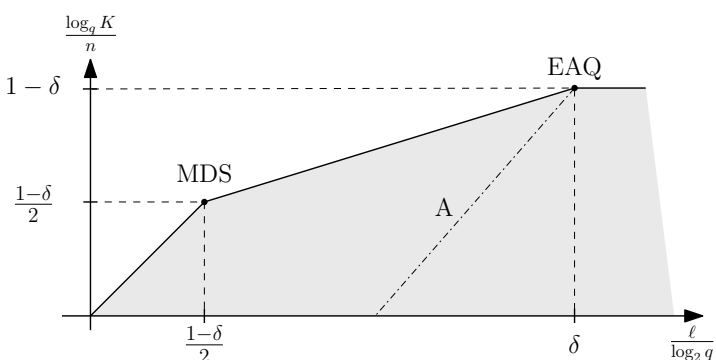

(c) $\delta>\frac{1}{2}$

Fig. 3. The rate region showing the admissible pairs of $\log _{2} K$ and $\ell$ (shown normalized with $n \log _{2} q$ ), according to the minimum distance. There is a qualitative difference between the cases $\delta=\frac{d-1}{n}<\frac{1}{2}$ (a) and $\delta=\frac{1}{2}$ (b) on the one hand, and $\delta>\frac{1}{2}$ (c) on the other. The maximum qubit rate is always $1-\delta$, attained with ebit rate $\delta$, in the former two cases that point (marked EAQ) contains the whole story, since moving along a line with slope 1 to the left, converting qubits to ebits, we get to the maximum unassisted rate $1-2 \delta$, and the maximum entanglement generation rate $-(1-2 \delta)$. (The negative sign is due to our convention of interpreting $E$ as the net entanglement consumption, which is a straightforward cost if $E \geq 0$, and generation of $-E$ ebits of entanglement if $E<0$ [16].) In the latter case, the boundary develops a kink at $\frac{1-\delta}{2}$ (point MDS) and becomes piecewise linear. The kink marks the change from Ineq. (30) being tight to Ineq. (31) being tight. In the text we explain that the points EAQ and MDS are attained by concrete codes, at least when $q$ is sufficiently large. The line $A$ marks a previous "quantum Singleton bound" [9].

Plugging this into Eq. (34), we obtain

$$
\begin{aligned}
2 \log _{2} K & \leq S\left(X_{[n]}\right)+n \Delta \\
& \leq(n-d+1) \overline{\bar{\sigma}}-\left(-1+\frac{d-1}{2 d-2-n}\right) n \Delta \\
& =(n-d+1) \overline{\bar{\sigma}}-\frac{n-d+1}{2 d-2-n} n \Delta \\
& \leq(n-d+1) \overline{\bar{\sigma}}-\frac{n-d+1}{2 d-2-n}\left(\log _{2} K-\ell\right),
\end{aligned}
$$

where the last line follows from Eq. (32). Solving for $\log _{2} K$ and simplifying leads to Eq. (28).

It remains to show that the same bounds hold for general encoders, even with nontrivial $F$ system. We do so by reducing it to the case of trivial $F$. Namely, consider any rank-one measurement $\left\{M_{t}\right\}$ on $F$, and define measurement probabilities $p_{t}$ and post-measurement states by letting $p_{t} \psi_{t}^{R A_{f} X_{J} X_{I} B_{i n}}:=\operatorname{Tr}_{F} \psi^{R A_{f} F X_{J} X_{I} B_{\text {in }}}\left(M_{t}^{F} \otimes \mathbb{1}\right)$ (Note that $\psi_{t}=\left|\psi_{t}\right\rangle\left\langle\psi_{t}\right|$ denotes the density matrix of the pure state $\left.\left|\psi_{t}\right\rangle\right)$. Ignoring the measurement outcome $t$, we obtain the following ensemble of pure states

$$
\psi^{R A_{f} X_{J} X_{I} B_{i n}}=\sum_{t} p_{t} \psi_{t}^{R A_{f} X_{J} X_{I} B_{i n}} .
$$

The decoder $V^{\prime}$ will work for all states $\psi_{t}$, resulting in the same pure states $\Phi_{K}^{R \widehat{M}}$ (maximally entangled) and $\varphi^{\prime A_{f} B_{f}}$ : indeed, $I\left(F: R A_{f}\right)=0$. In other words, $F$ is decoupled from $R A_{f}$, which means that conditional on $t$, the state of $R A_{f}$ is the same as the original $\psi^{R A_{f}}$.

An important consequence of this is that $\psi_{t}^{R}=\psi^{R}=$ $\frac{1}{K} \mathbb{1}_{R}$ is the maximally mixed state for all $t$. This in turn implies that there exists states $\left|\varphi_{t}\right\rangle^{A_{i n} B_{i n}}$ and isometries $V_{t}: M A_{i n} \hookrightarrow X_{[n]} A_{f}$ such that

$$
\left|\psi_{t}\right\rangle^{R A_{f} X_{J} X_{I} B_{i n}}=\left(V_{t} \otimes \mathbb{1}_{R B_{i n}}\right)\left(\left|\Phi_{K}\right\rangle^{R M}\left|\varphi_{t}\right\rangle^{A_{i n} B_{i n}}\right) .
$$

In other words, we have got an EAQEC for each $t$, consisting of the encoding and decoding isometries $V_{t}$ and $V^{\prime}$, respectively, and with initial and final entangled states $\varphi_{t}$ and $\varphi^{\prime}$, respectively. For a given $t$, such a code has the following properties: it transmits $\log _{2} K$ qubits as the original code, and it has a net entanglement consumption of $\ell_{t}=S\left(B_{i n}\right)_{\varphi_{t}}-S\left(A_{f}\right)_{\varphi^{\prime}}=S\left(B_{i n}\right)_{\psi_{t}}-$ $S\left(A_{f}\right)_{\psi_{t}}$. By Eq. (36), we have $\sum_{t} p_{t} \ell_{t} \leq \ell$, where we used concavity of the von Neumann entropy (note that $t$ only affects the initial, but not the final entanglement).

At the same time, the above proof shows that for each $t$, we have the bounds (24)-(28) for $\log _{2} K$ and $\ell_{t}$, with average entropies $\bar{\sigma}_{t}=\frac{1}{d-1} \mathbb{E}_{|J|=d-1} S\left(X_{J}\right)_{\psi_{t}}$ and $\overline{\bar{\sigma}}_{t}=$ $\frac{1}{n-d+a} \mathbb{E}_{|I|=n-d+1} S\left(X_{I}\right)_{\psi_{t}}$ on the right hand side. Once more by Eq. (36) and concavity of the entropy, we have $\sum_{t} p_{t} \bar{\sigma}_{t} \leq \bar{\sigma}$ and $\sum_{t} p_{t} \overline{\bar{\sigma}}_{t} \leq \overline{\bar{\sigma}}$, concluding the proof.

A code that has extremal parameters with respect to the quantum Singleton bound of Eqs. (29)-(31) in Theorem 6 is called entanglement-assisted quantum minimum distance separable (EAQMDS). The parameters of these codes are on the upper boundary of the regions in Fig. 3 (a)-(c).

We recall that for codes with no entanglement assistance the bound $d<\frac{n}{2}+1$ holds due to a no-cloning argument. Eq. (29) then yields the usual quantum Singleton bound for QECC, $\log _{2} K \leq(n-2 d+2) \log _{2} q$. As shown in Corollary 4, a QMDS code meeting this bound with equality must be pure. Next we show that this is no longer the case for EAQMDS codes. Recall that a quantum code is pure if all $(d-1)$-body marginals are maximally mixed. We make the assumption that the auxiliary output system $F$ of the encoder $V_{\text {ENC }}$ in Fig. 1 is trivial, as we have seen in the proof of Theorem 6 that the bound is independent of the dimension of $F$. We include, however, the situation of catalytic codes, i. e., the code might produce entanglement between the output $A_{f}$ and $B_{f}$.

Theorem 7 (Singleton bound for pure codes) Consider a pure EAQECC with minimum distance $d$ that encodes a $K$ dimensional quantum system into $n$ q-ary quantum systems 
and whose net entanglement consumption is $\ell$ ebits. Then

$$
\log _{2} K \leq(n-2 d+2) \log _{2} q+\ell .
$$

Proof: Consider the state $|\psi\rangle^{R A_{f} F X_{J} X_{I} B_{i n}}$ in Fig. 1. As we assume that the auxiliary output system $F$ is trivial, it can be omitted. Similar as in the derivation of Eqs. (14) and (15) in the proof of Theorem 5, the existence of a decoding isometry $V_{\mathrm{DEC}}^{\prime}$ implies that

$$
S\left(X_{I} B_{i n}\right)=S\left(X_{J}\right)+S\left(A_{f}\right)+S(R) .
$$

Hence

$$
\begin{aligned}
\log _{2} K & =S(R)=S\left(X_{I} B_{i n}\right)-S\left(X_{J}\right)-S\left(A_{f}\right) \\
& \leq S\left(X_{I}\right)+S\left(B_{i n}\right)-S\left(A_{f}\right)-S\left(X_{J}\right) \\
& \leq(n-d+1) \log _{2} q+\ell-(d-1) \log _{2} q .
\end{aligned}
$$

Here we have used subadditivity and the fact that for pure codes the reduced state on the subsystem $X_{J}$ is maximally mixed.

We note that a related result for the special case of nondegenerate stabilizer codes has recently been derived in [17, Thm. 10].

Corollary 8 An EAQMDS code $\mathcal{Q}$ with $d \geq \frac{n}{2}+1$ with net entanglement consumption $0<\ell<(d-1) \log _{2} q$ is not pure.

Proof: For $d \geq \frac{n}{2}+1$, the parameters of an EAQMDS code are on the upper boundary of the region in Fig. 3 (c). By Theorem 7, for pure codes the bound indicated by the line $A$ applies. The bound for pure codes only agrees with the general bound for $\ell=0$ and at the point marked EAQ with $\ell=(d-1) \log _{2} q$.

\section{OptimAl CONSTRUCTIONS}

We now turn to a discussion of what we know about the tightness of the various bounds derived in the preceding sections, depending on whether $d-1<\frac{n}{2}$ or $d-1 \geq \frac{n}{2}$. For this purpose we use the coding theory convention to read the logarithm in the formulas to base $q$, and we will be dealing with codes of $n$ qudits (systems of dimension $q$ ), and encoding a space of dimension $K=q^{k}$. We use the usual notation $((n, K, d))_{q}$ for a code of dimension $K$ with minimum distance at least $d$ on $n q$-ary systems; when $K=q^{k}$, this is equivalent to $\llbracket n, k, d \rrbracket_{q}$. For an EAQECC with code dimension $K=q^{k}$ and minimum distance $d$ that has a net consumption of $c=c_{i n}-c_{f}$ maximally entangled pairs of qudits, we use the notation $\llbracket n, k, d ; c \rrbracket_{q}$ (see [8]). Note that the net entanglement consumption is $\ell=c \log _{2} q$ ebits. For the convenience of the reader, we rephrase Theorem 6 in this coding theoretic context.

Corollary 9 Consider an EAQECC $\llbracket n, k, d ; c \rrbracket_{q}$ with minimum distance $d$ that encodes $k$ qudits into $n$ qudits with a net consumption of $c$ maximally entangled pairs of qudits. Then the following holds:

$$
\begin{aligned}
& k \leq c+\max \{0, n-2 d+2\}, \\
& k \leq n-d+1, \\
& k \leq \frac{(n-d+1)(c+2 d-2-n)}{3 d-3-n} \quad \text { if } d-1 \geq \frac{n}{2} .
\end{aligned}
$$

For all block lengths $n$ and $d-1 \leq \frac{n}{2}$, there exist QMDS codes with the parameters $\llbracket n, n-2 d+2, d \rrbracket_{q}$ if only the alphabet size $q$ is chosen large enough (see e.g. Ref. [7] for an overview). Naturally, then $c=0$, and in fact $c_{i n}=$ $c_{f}=0$. This construction achieves the point QMDS in Fig. 3 (a).

We note that for $d-1<\frac{n}{2}$, using linear programming the bound (39) has been derived in [18] for qubits; the generalization to prime powers $q$ is presented in [19].

Consider now the general case, including that of $d-1>\frac{n}{2}$. For all $n, d$, and large enough $q$, there exist entanglement-assisted quantum MDS (EAQMDS) codes with parameters $\llbracket n, n-d+1, d ; d-1 \rrbracket_{q}$; here $c_{i n}=d-1$ and $c_{f}=0$. This follows from the straightforward generalization of [20, Cor. 2] to prime power alphabets and the fact that for alphabet size $q^{2} \geq 9$, any linear code is equivalent to a code that trivially intersects its Hermitian dual code [21]. These codes correspond to the point marked EAQ in Fig. 3 (a)-(c). The line descending from that point to the lower left at slope 1 in Fig. 3 (a) and (b) is achievable by decreasing $k$ and simultaneously increasing $c_{f}$ by the same amount. In other words, we have the propagation rule $\llbracket n, k, d ; c \rrbracket_{q} \longrightarrow \llbracket n, k-1, d ; c-1 \rrbracket_{q}$. To see this, use the $\llbracket n, k, d ; c \rrbracket_{q}$ code to encode $k-1$ qudits as well as a maximally entangled state $\left|\Phi_{q}\right\rangle$; its decoder will recover the $k-1$ qudits, $c_{f}$ maximally pairs of maximally entangled pairs of qudits, plus another maximally entangled state $\left|\Phi_{q}\right\rangle$. This additional maximally entangled pair of qudits reduces the net consumption of maximally entangled pairs to $c-1$.

Somewhat surprisingly, any pure quantum code can be used to construct an EAQECC.

Theorem 10 Let $\mathcal{Q}$ be a pure QECC with parameters $\llbracket n, k, d \rrbracket_{q}$. Then EAQECC codes with parameters $\llbracket n-c, k, d ; c \rrbracket_{q}$ exist for all $c<d$.

Proof: Assume that we have a pure code $\mathcal{Q}=$ $((n, K, d))_{q}$ with an encoding isometry $V: M \rightarrow A_{f} F X_{[n]}$. We input half of a maximally entangled state $\left|\Phi_{K}\right\rangle^{R M}$ and obtain a pure state on $R A_{f} F X_{[n]}$. We split $X_{[n]}$ into two systems $X_{[n-c]}$ and $B_{i n}$ of size $n-c$ and $c<d$, respectively. As the code is pure and has minimum distance $d>c$, the reduced state on $B_{i n}$ is maximally mixed. Consider the Schmidt decomposition

$$
\begin{aligned}
V^{M}\left|\Phi_{K}\right\rangle^{R M} & =|\psi\rangle^{R A_{f} F X_{[n-c] B_{i n}}} \\
& =\frac{1}{\sqrt{q^{c}}} \sum_{i=1}^{q^{c}}\left|\psi_{i}\right\rangle^{R A_{f} F X_{[n-c]}}\left|\widetilde{\psi}_{i}\right\rangle^{B_{i n}},
\end{aligned}
$$


where the states $\left|\widetilde{\psi}_{i}\right\rangle$ form an orthonormal basis of the system $B_{i n}$. Hence there is a unitary transformation $U$ on $B_{\text {in }}$ that maps the standard basis on $B_{\text {in }}$ to this basis. Similarly, there is an isometry $W: M A_{\text {in }} \rightarrow A_{f} F X_{[n-c]}$ such that

$$
\begin{array}{r}
\left(W^{M A_{i n}} \otimes U^{B_{i n}}\right)\left(\left|\Phi_{K}\right\rangle^{R M}|\varphi\rangle^{A_{i n} B_{i n}}\right) \\
=\frac{1}{\sqrt{q^{c}}} \sum_{i=1}^{q^{c}}\left|\psi_{i}\right\rangle^{R A_{f} F X_{[n-c]}\left|\widetilde{\psi}_{i}\right\rangle^{B_{i n}}},
\end{array}
$$

where $|\varphi\rangle^{A_{\text {in }} B_{\text {in }}}$ is a maximally entangled state.

As $U$ acts only on $B_{i n}$, it can be applied by the receiver, followed by the decoding isometry for the original code. That shows that the minimum distance of the new code is at least $d$.

This propagation rule generalizes that from Ref. [22] for pure qubit stabilizer codes to arbitrary pure codes, and corrects and generalizes the original propagation rule from Ref. [23] that would violate the EAQEC Singleton bound [Theorem 6] when starting with a stabilizer QMDS code (see also Ref. [24]).

Corollary 11 Any QMDS code with parameters $\llbracket n, n-2 d+$ $2, d \rrbracket_{q}$ gives rise to an EAQMDS code with the parameters $\llbracket n-c, n-2 d+2, d ; c \rrbracket_{q}$ for all $c<d$.

Proof: Proposition 4 states that all QMDS codes are pure. The claim follows from Theorem 10.

This construction works in particular when starting from an absolutely maximally entangled (AME) state with an even number $n$ of parties. These are pure quantum states for which maximal entanglement is present across every bipartition. As with QMDS codes, such states always exist as long as the local dimension $q$ is chosen large enough. (For example, the CSS construction of a so-called Euclidean QMDS code requires $n \leq q+1$ for $2<q$.) For an even number $n$ of parties, AME states are QMDS codes with parameters $\llbracket n, 0, \frac{n}{2}+1 \rrbracket_{q}$. With the propagation rule for unassisted pure codes $\left(\llbracket n, k, d \rrbracket_{q} \longrightarrow \llbracket n-1, k+1, d-1 \rrbracket_{q}\right.$, c.f. Refs. [25] and [7]) one obtains $\llbracket n-k, k, \frac{n}{2}+1-k \rrbracket_{q}$ QMDS codes. With Corollary 11 this yields EAQMDS codes with parameters $\llbracket n-k-t, k, \frac{n}{2}+1-k ; t \rrbracket_{q}$ for $n+2=k+2 d$ and $\frac{n}{2}+1-k>t$. (See [7] for the discussion of other propagation rules within the class of unassisted QECC.) This shows once more that the point EAQ in Fig. 3 (a)-(c) is attained. Nevertheless, the whole set of QMDS codes is in a sense strictly more powerful in this construction than when one considers solely AME states. As an example, a QMDS code with parameters $\llbracket 8,4,3 \rrbracket_{3}$ can be used to yield a $\llbracket 7,4,3 ; 1 \rrbracket_{3}$ EAQMDS code. This cannot be realized with AME states: it has been shown that there are no AME states on $2 n-2 t=14-2=12$ qutrits, in other words, a $\llbracket 12,0,7 \rrbracket_{3}$ code does not exist [7].

Continuing with the case $d-1 \geq \frac{n}{2}$, we turn our attention to the point marked MDS in Fig. 3 (c) which has $k=c=\frac{1}{2}(n-d+1)$. It is also achievable: use a classical MDS code to encode $(n-d+1) \log _{2} q$ bits on $n$ quantum systems. These bits are used to teleport $\frac{1}{2}(n-d+1) \log _{2} q$ qubits, using $\frac{1}{2}(n-d+1) \log _{2} q$ ebits in entanglement assistance. This is the basic idea underlying the counterexamples in Ref. [14]. By simply using less information from the classical MDS code, and concomitantly less entanglement, we can clearly attain the entire straight line connecting the point MDS to the origin in (c).

It is unclear what the status of the line connecting the points MDS and EAQ in Fig. 3 (c) is; however, one might conjecture that it is "essentially" attainable for large enough alphabet size $q$, i. e. all integer points below it correspond to possible code values. This would for example follow if there exist hybrid codes that transmit certain numbers of classical bits and qubits assisted by entanglement, after which we convert the classical bits to qubits by consuming even more entanglement in teleportation. Concretely, it would be enough to find EAQECC $\llbracket n, k, d ; c \rrbracket_{q}$ with $k=\frac{n-d+1}{d-1} c$ that can simultaneously transmit $j=\frac{n-d+1}{d-1}(d-1-c)$ classical bits. Note that in this case, $j+k=n-d+1$, which is the relation for the parameters of a classical MDS code.

What about other propagation rules, for example is there a chance to turn any $\llbracket n, k+c, d ; c \rrbracket_{q}$ into an $\llbracket n, k, d \rrbracket_{q}$ ? This does not seem to be the case in general: for example, a $\llbracket 4,1,3 ; 1 \rrbracket_{2}$ EAQMDS code has been constructed [8], while it has been shown in Ref. [26] that no $\llbracket 4,0,3 \rrbracket_{2}$ exists. It is worth pointing out that the propagation rule described at the beginning of this section yields a $\llbracket 4,0,3 ; 0 \rrbracket_{2}$ EAQECC, which is no contradiction, as it has $c_{i n}=c_{f}=1$ rather than 0 .

\section{DISCUSSION}

The bounds from Theorem 6 give precisely the qubitebit capacity region of the erasure channel, for erasure probability $\delta \in[0,1]$, and for any alphabet size $q$ [27]. (We remark that the treatment in [27] seems implicitly to have assumed $\delta<\frac{1}{2}$, though.)

In particular, the region is not just an outer bound but several lines and isolated points of it are also attainable by codes of sufficiently large alphabet size $q$. This is no coincidence, as the Singleton bound really is about the capability of a code to correct erasure errors. Furthermore, the consistent use of the von Neumann entropy in the proofs makes the bounds robust to small deviations from the ideal. That is, they will essentially still hold even if the decoder works only for most of the erasure pattern subsets $J$ of size $d-1$, and only decodes with fidelity close to 1 . This is because the decoupling condition for QECC will be replaced by a trace distance bound of $\epsilon$ between the actual state and the decoupled state [10], and this results in Eq. (4), and analogously in later proofs, being replaced by an approximate equality, up to terms $O\left(\epsilon n \log _{2} q+h(\epsilon)\right)$, by the Fannes continuity inequality for the von Neumann entropy and conditional entropy [28]-[30]. The rest of the proof follows unchanged. 
There are many open problems left, the first being the attainability of the bounds along the line MDS-EAQ when $d-1>\frac{n}{2}$, at least for sufficiently large alphabet size $q$. We know that the endpoints are attained in this way, and so we are asking for some sort of interpolation between the two corresponding constructions. Note that our bounds cut out a convex region, but a priori there is no reason why the actually achievable codes and their qubit-ebit tradeoff should have any sort of convexity property. Returning briefly to the Shannon theoretic model of only demanding high-fidelity decoding for most erasure patterns, though, we observe that there at least the convexity is essentially granted: This is because we can juxtapose codes of block length $n_{1}$ and of block length $n_{2}$ for the same relative distance $\delta$, and get one that corrects with high fidelity for most erasures, at marginally smaller relative distance $\delta-\epsilon$ of block length $n_{1}+n_{2}$, while the code lengths add.

A second important question is, what are the special properties characterizing the codes attaining the bounds?

Third, what are the constraints on the alphabet size $q$ for an EAQMDS code $\llbracket n, k, d ; c \rrbracket_{q}$ to exist? For QMDS codes is it known that for $d \geq 3$ the alphabet size must satisfy $n \leq q^{2}+d-2$ [7], and it is reasonable to expect that a similar scaling should hold for EAQMDS.

Finally, how do these bounds compare to previous algebraic quantum Singleton-type bounds, and in particular to the recent results of Lai and Ashikhmin [18] for EAQECC?

\section{ACKNOWLEDGMENTS}

We thank Andrew Nemec as well as the anonymous reviewers for valuable comments on earlier versions of the manuscript. Questions by one of the latter led to the formulation of Theorem 7 .

MG acknowledges support by the Foundation for Polish Science (IRAP project, ICTQT, contract no. 2018/MAB/5, co-financed by EU within Smart Growth Operational Programme).

FH was supported by the Fundació Cellex, the Spanish MINECO (projects QIBEQI FIS2016-80773-P and Severo Ochoa SEV-2015-0522), the Generalitat de Catalunya (SGR-1381 and CERCA Programme), the European Union under Horizon2020 (PROBIST 754510), and the Foundation for Polish Science through TEAMNET (POIR.04.04.00-00-17C1/18-00).

AW acknowledges financial support by the Spanish MINECO (projects FIS2016-86681-P and PID2019107609GB-I00/AEI/10.13039/501100011033) with the support of FEDER funds, and the Generalitat de Catalunya (project 2017-SGR-1127).

\section{REFERENCES}

[1] Markus Grassl, Thomas Beth, and Thomas Pellizzari, "Codes for the quantum erasure channel", Phys. Rev. A 56(1):33-38, 1997.

[2] Richard C. Singleton, "Maximum distance $q$-nary codes", IEEE Trans. Inf. Theory 10(2):116-118, 1964.
[3] Emanuel Knill and Raymond Laflamme, "A theory of quantum error-correcting codes", Phys. Rev. A 55(2):900-911, 1997.

[4] Eric M. Rains, "Nonbinary Quantum Codes", IEEE Trans. Inf. Theory 45(6):1827-1832, 1999.

[5] Andreas Klappenecker and Pradeep K. Sarvepalli, "On subsystem codes beating the quantum Hamming or Singleton bound", Proc. Roy. Soc. London A 463(2087):2887-2905, 2007.

[6] Charles H. Bennett, David P. DiVincenzo, John A. Smolin, and William K. Wootters, "Mixed-state entanglement and quantum error correction", Phys. Rev. A 54(5):3824-3851, 1996.

[7] Felix Huber and Markus Grassl, "Quantum Codes of Maximal Distance and Highly Entangled Subspaces", Quantum 4:284, 2020.

[8] Todd A. Brun, Igor Devetak and Min-Hsiu Hsieh, "Correcting quantum errors with entanglement", Science 314(5798):436-439, 2006.

[9] Todd A. Brun, Igor Devetak and Min-Hsiu Hsieh, "Catalytic quantum error correction", IEEE Trans. Inf. Theory 60(6):3073-3089, 2014.

[10] Benjamin Schumacher and Michael D. Westmoreland, "Approximate Quantum Error Correction", Quantum Inf. Proc. 1(1+2):5-12, 2002.

[11] Dorit Aharonov, Michael Ben-Or, Russell Impagliazzo and Noam Nisan, "Limitations of Noisy Reversible Computation", arXiv:quant-ph/9611028, 1996.

[12] Marius Junge and Carlos Palazuelos, "CB-norm estimates for maps between non-commutative $L_{p}$-spaces and quantum channel theory", arXiv:1407.7684 [math.OA], 2014.

[13] Alexander Müller-Hermes, Daniel Stilck França and Michael M. Wolf, "Relative Entropy Convergence for Depolarizing Channels", arXiv:1508.07021 [quant-ph], 2015.

[14] Markus Grassl, "Entanglement-assisted quantum communication beating the quantum Singleton bound", talk at AQIS 2016, Taiwan.

[15] Markus Grassl, "Entanglement-Assisted Quantum Communication Beating the Quantum Singleton Bound", Phys. Rev. A 103(2):020601, 2021.

[16] Igor Devetak, Aram W. Harrow and Andreas Winter, "A Resource Framework for Quantum Shannon Theory", IEEE Trans. Inf. Theory 54(10):4587-4618, 2008

[17] Priya J. Nadkarni and Shayan Srinivasa Garani, "Nonbinary Entanglement-assisted Stabilizer Codes", Quant. Inf. Proc. 20(8):256, 2021

[18] Ching-Yi Lai and Alexei Ashikhmin, "Linear Programming Bounds for Entanglement-Assisted Quantum Error-Correcting Codes by Split Weight Enumerators", IEEE Trans. Inf. Theory 64(1):622-639, 2018.

[19] A. Allahmadi, A. AlKenani, R. Hijazi, N.Muthana, F. Özbudak and P. Solé, "'New constructions of entanglement-assisted quantum codes", Cryptogr. Commun., 2021.

[20] Mark M. Wilde and Todd. A. Brun, "Optimal entanglement formulas for entanglement-assisted quantum coding", Phys. Rev. A 77(6):064302, 2008

[21] Claude Carlet, Sihem Mesnager, Chunming Tang and Ruud Pellikaan, "Linear Codes Over $\mathbb{F}_{q}$ Are Equivalent to LCD Codes for $q>3$ ", IEEE Trans. Inf. Theory 44(4):3010-3017, 2018.

[22] Ching-Yi Lai and Todd A. Brun, "Entanglement-assisted quantum error-correcting codes with imperfect ebits", Phys. Rev. A 86(3):032319, 2012

[23] Carlos Galindo, Fernando Hernando, Ryutaroh Matsumoto and Diego Ruano, "Entanglement-assisted quantum error-correcting codes over arbitrary finite fields", Quant. Inf. Proc. 18(4):116, 2019. See [24] for corrections.

[24] Carlos Galindo, Fernando Hernando, Ryutaroh Matsumoto and Diego Ruano, "Correction to: Entanglement-assisted quantum error-correcting codes over arbitrary finite fields", Quant. Inf. Proc. 20(6):216, 2021

[25] Eric M. Rains, "Quantum weight enumerators", IEEE Trans. Inf. Theory 44(4): 1388-1394, 1998.

[26] Atsushi Higuchi and Anthony Sudbery, "How entangled can two couples get?", Phys. Lett. A 273(4):213-217, 2000.

[27] Min-Hsiu Hsieh and Mark M. Wilde, "Entanglement-Assisted Communication of Classical and Quantum Information", IEEE Trans. Inf. Theory 56(9):4682-4704, 2001.

[28] Mark Fannes, "A continuity property of the entropy density for spin lattice systems", Commun. Math. Phys. 31(4):291-294, 1973. 
[29] Koenraad M. R. Audenaert, "A sharp continuity estimate for the von Neumann entropy", J. Phys. A Math. Theor. 40(28):8127-8136, 2007.

[30] Andreas Winter, "Tight Uniform Continuity Bounds for Quantum Entropies: Conditional Entropy, Relative Entropy Distance and Energy Constraints", Commun. Math. Phys. 347(1):291-313, 2016.

Markus Grassl (S'94-M'00-SM'20) received his diploma degree in Computer Science in 1994 and his doctoral degree in 2001, both from the Fakultät für Informatik, Universität Karlsruhe (TH), Germany. From 1994 to 2007 he was a member of the Institut für Algorithmen und Kognitive Systeme, Fakultät für Informatik, Universität Karlsruhe (TH), Germany.

From 2007 to 2008 he was with the Institute for Quantum Optics and Quantum Information of the Austrian Academy of Sciences in Innsbruck. From 2009 to 2014, he was a Senior Research Fellow at the Centre for Quantum Technologies at the National University of Singapore. In 2014, he joined the Friedrich-Alexander-Universität ErlangenNürnberg and the Max Planck Institute for the Science of Light (MPL) Erlangen. Since 2019, he is a Senior Scientist at the International Centre for Theory of Quantum Technologies, University of Gdansk. His research interests include quantum computation, focusing on quantum error-correcting codes, and methods of computer algebra in algebraic coding theory. He maintains tables of good block quantum errorcorrecting codes as well as good linear block codes.

Dr. Grassl served as Associate Editor for Quantum Information Theory of the IEEE Transactions on Information Theory from 2015 till 2017.
Felix Huber received a M.S. degree in physics from ETH Zürich, Switzerland, in 2012, and a Ph.D. degree in theoretical quantum optics from the Universität Siegen, Germany, in 2017.

In 2018 he was a Postdoctoral Researcher at the Universität $\mathrm{zu}$ Köln, Germany, and from 2018 to 2020 he was a Research Fellow at ICFO Barcelona, Spain. Since 2021 he is an Adiunkt at the Uniwersytet Jagielloński in Kraków, Poland. His research interests include quantum error correction, multipartite entanglement, and invariant theory.

Dr. Huber is recipient of the 2019 Dissertation Prize by the section AMOP of the German Physical Society.

Andreas Winter received a Diploma degree in Mathematics from the Freie Universität Berlin, Germany, in 1997, and a Ph.D. degree from the Fakultät für Mathematik, Universität Bielefeld, Germany, in 1999.

He was Research Associate at the University of Bielefeld until 2001, and then with the Department of Computer Science at the University of Bristol, UK. In 2003, still with the University of Bristol, he was appointed Lecturer in Mathematics, and in 2006 Professor of Physics of Information. Since 2012 he has been ICREA Research Professor with the Universitat Autònoma de Barcelona, Spain. His research interests include quantum and classical Shannon theory, and discrete mathematics.

Prof. Winter is recipient, along with Charles H. Bennett, Igor Devetak, Aram W. Harrow and Peter W. Shor, of the 2017 Information Theory Society Paper Award. 